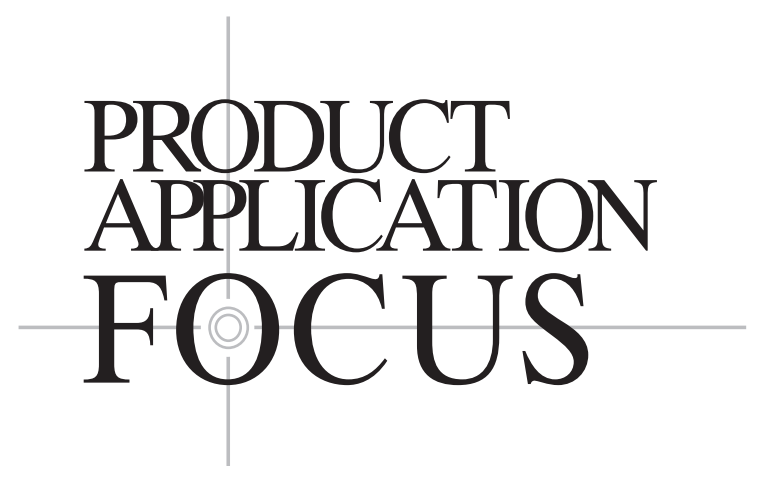

\title{
Small-Scale Telomere Repeat Sequence Content Assay Using Pyrophosphorolysis Coupled with ATP Detection
}

\author{
Randall D. Learish, John Shultz, Samuel Ho, and Robert F. Bulleit \\ Promega, Madison, WI, USA
}

BioTechniques 33:1349-1353 (December 2002)

\begin{abstract}
Studies of telomere length have been carried out in diverse areas of research. However, current methods to measure telomeres are cumbersome and not amenable to high-throughput analyses. Using a coupled pyrophosphorolysis/trans-phosphorylation reaction, we have developed a novel assay to quantitate telomere sequence content in a single tube or 96-well format. The method uses a telomerespecific oligonucleotide probe to sample nanogram quantities of DNA without PCR amplification. Polymerase and kinase enzymes drive the production of ATP, which is then monitored with a luciferase enzyme reporter system. Using this approach, we demonstrated that the luminescent output was linear across a 100-fold range of DNA input, and the assay was sensitive to 0.4-1 ng DNA. A control probe reaction and a DNA quantitation reaction were also designed using the same pyrophosphorolysis technology to correct for background activity and normalize the signal against variations in DNA input, respectively. Finally, we show that the normalized luminescent signal generated by this new method is highly correlated to the telomere restriction fragment length for six human cell lines.
\end{abstract}

\section{INTRODUCTION}

Telomeres are highly repetitive DNA sequences at the distal ends of chromosomes that vary in length and function and maintain genome integrity $(1,6)$. The vertebrate telomere is composed of a highly abundant six-base repeat (TTAGGG) that is a target for the hybridization of oligonucleotide probes in a variety of assays. Telemere restriction fragment length (TRFL) analyses employ Southern blot methodologies to assign a mean telomere length estimate to restriction-digested DNA obtained from a pool of cells. To determine the TRFL, the integration of the hybridizing signal is carried out across each lane and compared to the position of molecular weight markers. Quantitative fluorescent in situ hybridization (FISH) measures the abundance of fluorescently labeled telomere probes on spreads of mitotic chromosomes and is currently the only method that permits the study of telomere dynamics on specific chromosomes (7). A variation of this method, termed flow-FISH, involves probe hybridization within fixed, permeabilized cells. The labeled cells are passed through a flow cytometer and the abundance of the repeat sequence (i.e., the level of fluorescence) is compared to a control cell line (5). However, limitations to each of these methods have hampered telomere research. TRFL analysis requires the careful preparation and digestion of microgram quantities of DNA and is time consuming and cumbersome in terms of equipment and solution handling. FISH-based systems require expensive equipment, a high degree of technical expertise, and a significant amount of time. Furthermore, the number of samples that may be processed is small. Here we describe the development of a novel telomere assay to measure global changes in telomere sequence content. The system involves measuring ATP production from a pyrophosphorolysis/trans-phosphorlyation reaction initiated by a hybridization event $(12,13)$. This assay offers a level of sensitivity and speed that is a significant improvement over existing telomere assays.

\section{MATERIALS AND METHODS}

Telo-Glo $^{\mathrm{TM}}$ is an application of READIT ${ }^{\mathrm{TM}}$ technology (Promega, Madison, WI, USA) $(12,13)$. The following components are from Promega and comprise the new assay: denaturation solution, neutralization solution, a telomere-specific oligonucleotide (telo-probe), and an oligonucleotide identical to the telo-probe except for four mismatches in the terminal five 
bases at the $3^{\prime}$-end (control probe). Oligonucleotides were synthesized by standard methods, HPLC purified, lyophilized, and redissolved in neutralization buffer at $100 \mu \mathrm{M}$. Also included was a restriction enzyme mixture $(5 \mathrm{U} / \mu \mathrm{L})$ that contains frequent-cutting enzymes that generate $5^{\prime}$ overhangs, thermostable TeloDetection Enzymes ${ }^{\mathrm{TM}}$, which is a mixture of READase $^{\mathrm{TM}}$ polymerase (a DNA polymerase), READase kinase (a nucleotide kinase), $\mathrm{ADP}, \mathrm{MgCl}_{2}$, and sodium pyrophosphate $(12,13)$, ENLITEN ${ }^{\circledR}$, a lyophilized luciferin/luciferase detection reagent, and ENLITEN reconstitution buffer.

Reactions using the telo-probe and control probes were prepared in an identical manner, except for the use of the oligonucleotide. Genomic DNA was diluted to $1 \mathrm{ng} / \mu \mathrm{L}$, and $10 \mu \mathrm{L}$ aliquots were arrayed into $0.5-\mathrm{mL}$ reaction tubes or $96-$ well plates. Ten microliters of denaturing solution were added, and the samples were incubated at room temperature for $15 \mathrm{~min}$. An enzyme master mixture was prepared by combining TeloDetection enzymes, neutralization solution, and the appropriate probe in a ratio of 10:9:1. Subsequently, 40 $\mu \mathrm{L}$ master mixture were added to each denatured sample. For the DNA quantitation reaction, the DNA was diluted to 50 $\mathrm{pg} / \mu \mathrm{L}$, and $10 \mu \mathrm{L}$ aliquots were arrayed into $0.5-\mathrm{mL}$ reaction tubes or 96-well plates, the denaturation and neutralization solutions were premixed in a $1: 2$ ratio, and then $10 \mu \mathrm{L}$ of this "mock" denaturing solution were added. This prevented the DNA from being denatured and from the loss of restriction enzyme sites. A master mixture was prepared by combining the TeloDetection enzymes, mock denaturation solution, and restriction enzymes in a ratio of 100:99:1. Forty microliters of this master mixture were added to each DNA sample to achieve a final volume of $60 \mu \mathrm{L}$. All reactions were preincubated at $37^{\circ} \mathrm{C}$ for $30 \mathrm{~min}$ to promote restriction enzyme activity. To initiate pyrophosphorolysis, the samples were subsequently incubated at $55^{\circ} \mathrm{C}$ for $60 \mathrm{~min}$. Single-tube reactions were incubated in a water bath, transferred to luminometer tubes, $50 \mu \mathrm{L}$ ENLITEN were added, and then the light output was read in the TD-20/20 luminometer (Turner Biosystems, Sunnyvale, CA, USA). For 96-well plates, the samples were incubated in a programmed thermal cycler (GeneAmp ${ }^{\circledR}$ PCR System 9600; Applied Biosystems, Foster City, CA, USA), transferred to an opaque plate (Clini-plate; Robbins Scientific, Sunnyvale, CA, USA), $50 \mu$ L ENLITEN were added, and the readout was performed using the Reporter Microplate Reader (Turner Biosystems) or the Microlumat Plus LB96V Luminometer (Berthold Technologies, Pforzheim, Germany). The numerical output was dependant on the gain settings for each instrument, the data below are thus represented as relative light units (RLU).

\section{Cell Lines}

The following human cell lines were stocks originally derived from ATCC (Manassas, VA, USA): promyelocytic leukemia (HL60), embryonic kidney (HEK293), neuroblastoma (SH-SY5Y), T-cell leukemia (Jurkat), and cervical carcinoma (HeLa). HeLa1.2.11 was obtained from T. DeLange and J. Karlseder (Rockefeller University, New York, NY, USA). Cells were propagated in DMEM/high-glucose culture media (Invitrogen, Carlsbad, CA, USA) containing glutamine and 10\% FBS (HyClone Laboratories, Logan, UT, USA). Cells were passaged at least twice and then harvested, washed with serum-free media, and pelleted by centrifugation at $350 \times \mathrm{g}$.

\section{DNA Preparation and Southern Blot Analysis}

Cell pellets were subjected to a standard phenol-chloroform extraction protocol, followed by ribonuclease treatment (11). DNA from canine, porcine, bovine, fruit fly (Drosophila melanogaster), rat (Sprague-Dawley $\left.{ }^{\circledR}\right)$, and mouse (Mus spretus) samples was obtained from BD Biosciences Clontech (Palo Alto, CA, USA). The species of non-murine vertebrate samples was unknown. Human mixed blood DNA, yeast (Saccharomyces cerevisiae), and $\lambda$ phage DNAs were obtained from Promega. The concentration and quality of each sample were obtained by spectrophotometric and gelbased analyses to confirm that the DNA was intact and substantially free of degradation. TRFL analysis was performed using the TeloTAGGG ${ }^{\mathrm{TM}}$ Telomere Length Assay kit (Roche Applied Science, Indianapolis, IN, USA). The blot was processed and exposed to film, a digital image was created, and each lane was then analyzed using the Telometric software as previously described (4).

\section{RESULTS}

DNA polymerases catalyze the incorporation of dNTPs to the $3^{\prime}$-end of a primer-template hybrid. This reaction involves the linkage of the $\gamma$ phosphate group of dNTP with the $3^{\prime}$ hydroxyl of the terminal ribose group on the growing DNA strand, and the release of $\mathrm{PP}_{\mathrm{i}}$.

$$
\mathrm{DNA}_{\mathrm{n}}+\mathrm{dNTP} \leftrightarrow \mathrm{DNA}_{\mathrm{n}+1}+\mathrm{PP}_{\mathrm{i}}
$$

Pyrophosphorolysis describes the reverse reaction-the cleavage of the terminal phosphodiester bond and the formation of high-energy dNTPs. High levels of $\mathrm{PP}_{\mathrm{i}}$ can drive this "de-polymerase" activity by mass action $(3,8,10)$. Because the telomere is highly conserved among vertebrates, we explored the idea that this technology might be adapted to quantitate telomere repeat sequence content by the use of specific probes. A cartoon depicts the assay design (Figure 1). For initial experiments, several parameters were adopted based on a previous DNA quantitation assay system (8), including the working concentration of DNA (10 ng), the concentration of the oligonucleotide used, and the incubation times. Also, it had been previously demonstrated that the neutralization, hybridization, pyrophosphorolysis, and kinase activities occur together in a single solution format.

To test the specificity of the proposed reaction, we designed and screened a variety of oligonucleotides based on the repeat $[\text { TTAGGG }]_{n}$ to identify a sequence that would yield the greatest ATP-producing activity against a template DNA sample from human blood. An 18-mer probe (teloprobe) was selected and used to study the time course of ATP accumulation using the pyrophosphorolysis reaction (Figure 2A). Little activity occurred during a 30 -min preincubation at $37^{\circ} \mathrm{C}$. However, during the $55^{\circ} \mathrm{C}$ incubation (a near-optimal temperature for the polymerase), ATP accumulated rapidly for $60 \mathrm{~min}$. Background activity in the absence of the probe (no probe) represented approximately $10 \%$ of the telo-probe signal. We adopted a fixed total incubation time of $90 \mathrm{~min}$ for subsequent reactions because, after that time point, ATP generation slowed due to accumulation of reaction products that interfere with the pyrophosphorolysis activity.

The telomeres of non-vertebrate species do not share significant homology with those of vertebrates. Thus, to test further 
the specificity of the telo-probe, we performed the reaction on a panel of vertebrate or non-vertebrate DNA samples. Figure 2B shows the telo-probe yielded a specific signal across six vertebrate species. We made no attempt to correlate the luminescent values to TRFL because the proper background subtraction was unavailable at this stage of assay development, and the species information on the DNA samples was limited. Nonetheless, it was noted that the signal generated against chicken DNA was highest, perhaps because chickens possess microsatellite DNA with highly abundant telomere-like repeat sequences (2). As predicted, in fruit flies, yeast, and $\lambda$ phage samples (non-vertebrates), the amount of activity was minimal. There was no correlation between the amount of "no probe" background activity and the amount of telo-probe signal.

To examine the sensitivity and linearity of the telo-probe reaction, we titrated varying amounts of human or chicken DNA into reactions with a fixed amount $(3.3 \mu \mathrm{M})$ of probe (Figure 2C). For the human sample, the sensitivity was approximately $1 \mathrm{ng}$, as determined by the fact that this amount of DNA produced a signal that was greater than two standard deviations from the level of background activity. The signal increased linearly with increasing DNA between 1 and 100 ng. For chicken DNA, the sensitivity was $0.4 \mathrm{ng}$, and the linear range was between 0.4 and $50 \mathrm{ng}$ DNA. Combined, Figure 2, panels $\mathrm{A}-\mathrm{C}$, indicates that the assay is specific and highly sensitive. However, it was also noted that the amount of background activity associated with different DNA samples varied. In some non-vertebrate samples (e.g., $\lambda$ phage) that do not possess the target, the telo-probe produced a small amount of activity. We reasoned that this might represent probe interactions with weakly homologous DNA targets or background activity contributed by the probe itself. Therefore, we sought to develop a control probe reaction.

For the DNA polymerase to initiate pyrophosphorolysis, the $3^{\prime}$ terminal nucleotide of the probe must be perfectly matched to its template. We predicted and subsequently demonstrated that the introduction of mismatching nu-

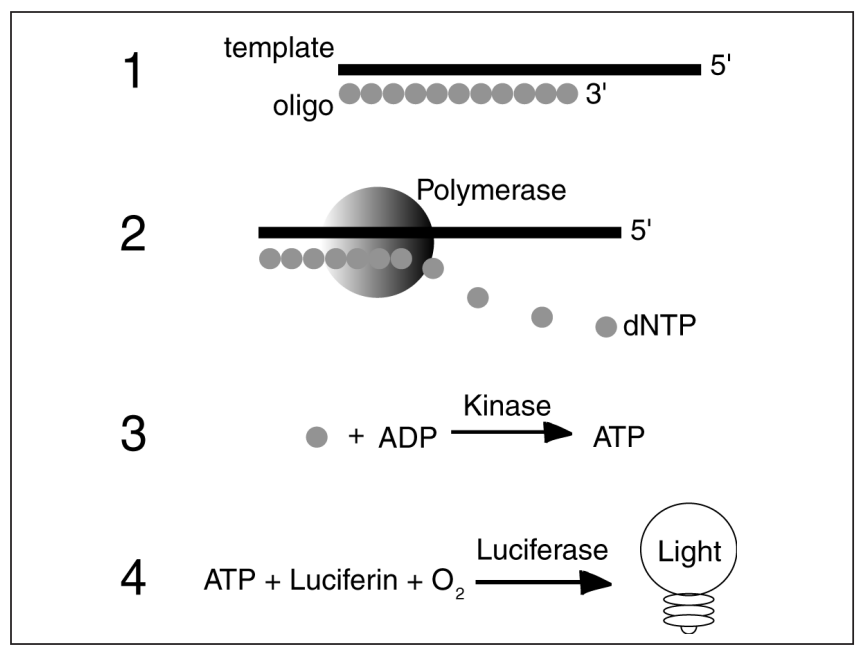

Figure 1. Telo-Glo Assay scheme. The reaction is initiated by the denaturation of genomic DNA (template), followed by hybridization with a telomerespecific oligonucleotide probe. Provided that the extreme $3^{\prime}$-end of the probe is perfectly matched, the hybrid becomes a substrate for polymerase binding, and pyrophosphorolysis is carried out in the presence of sodium $\mathrm{PP}_{\mathrm{i}}$, releasing high-energy dNTPs (circles). Next, the dNTPs are used as phosphate donors for the conversion of ADP to ATP by a nucleotide kinase. Finally, the reaction sequence is coupled to the luciferin-luciferase enzyme reporter system to quantitate the level of ATP produced. cleotides at the 3 '-end of the telo-probe would block pyrophosphorolysis. We selected one mismatched probe for use as a control because it produced activity against non-vertebrate DNA that was similar in magnitude to the activity generated by the telo-probe against the same DNA. Against the human DNA sample, the control probe generated $11.3 \%$ of the signal produced by the telo-probe (data not shown). The control probe signal subtracted from the telo-probe signal will be referred to as the "net telo-probe" signal.

To make comparative telomere measurements using the net telo-probe signal, one must assume that equal amounts of DNA input are achieved. A retrospective analysis of the samples shown in Figure 2B indicated that there were small discrepancies in the input amounts caused by variations associated with DNA quantitation and small volume handling of concentrated DNA. This limited our ability to compare the telomere content between samples. Therefore, we incorporated a means of normalizing the net telo-probe signal versus DNA input using an existing DNA quantitation assay based on the same pyrophosphorolysis technology (DNA Quantitation System; Promega). Using a modification of this system, we designed a third reaction component (DNA quantitation reaction) by preparing a separate enzyme master mixture that included the appropriate restriction enzymes but without oligonucleotides. This master mixture generates ATP because the DNA polymerase can initiate pyrophophorolysis not only from the $3^{\prime}$-end of a probe-template hybrid but also from terminal $5^{\prime}$ overhangs. We titrated varying amounts of the restriction enzyme mixture into a reaction with $500 \mathrm{pg}$ DNA under non-denaturing conditions in the absence of oligonucleotides. The samples were preincubated for $30 \mathrm{~min}$ at $37^{\circ} \mathrm{C}$ (the optimal temperature for restriction enzyme activity), and then the temperature was shifted to $55^{\circ} \mathrm{C}$ to initiate pyrophosphorolysis. The results showed a linear increase in light output with increasing amounts of restriction enzymes, and a maximal level of activity was reached at $1 \mathrm{U}$ per reaction (data not shown). Next, the sensitivity and linearity of the DNA quantitation reaction were examined by the titration of varying amounts of human genomic DNA into the assay with a fixed amount of restriction enzymes $(1 \mathrm{U})$. Figure $2 \mathrm{D}$ shows that the restriction enzymes were active within the master mixture, and the assay is sensitive to as little as $10 \mathrm{pg}$ DNA and linear in the range from $10 \mathrm{pg}$ to $1 \mathrm{ng}$. When the restriction enzyme was left out of the master mixture, only very low levels of activity were present. By combining the probe-based reactions and the DNA quantitation reactions, we derived the following ratio:

$$
\mathrm{T} / \mathrm{Q}=\frac{\left(\mathrm{RLU}_{\text {telo-probe }}-\mathrm{RLU}_{\text {Control probe }}\right)}{\left(\mathrm{RLU}_{\mathrm{DNAQ}}-\mathrm{RLU}_{\mathrm{H}_{2} \mathrm{O} \text { blank }}\right)}
$$

where T/Q represents the net telo-probe/DNA quantitation reaction, and DNAQ represents the DNA quantitation reaction. In this manner, the net telo-probe signal is normalized against DNA input.

Having defined the linear range for both the telo-probe reaction and the DNA quantitation reaction, we arrived at recommendations for DNA input for each reaction component simply by choosing amounts of DNA $-10 \mathrm{ng}$ and $500 \mathrm{pg}$, respectively - that fell in the middle of each linear range.

We obtained commercially available human cell lines and HeLa1.2.11, a previously characterized line known to have long telomeres (14) and isolated DNA from each cell line. A small portion of each DNA sample was diluted to $1 \mathrm{ng} / \mu \mathrm{L}$ for 
use in the telo-probe and control probe reactions and 50 $\mathrm{pg} / \mu \mathrm{L}$ for the DNA quantitation reaction. The concentrated DNA extracts were then processed for TRFL analysis (Figure 3A). Using digital imaging software, we obtained histograms representing telomere fragment sizes and statistical information including the mean, median, and modal TRFL for each sample (data not shown). Despite the appearance by eye that the smearing patterns in lanes 2-6 were normal distributions, the histograms suggested that each distribution was skewed toward smaller molecular weights. In addition, in the Jurkat sample (Figure 3, lane 1), a molecular species of $>21 \mathrm{~kb}$ was observed, and a second intensity peak appeared between the 7.4 and $6.1 \mathrm{~kb}$ markers, resulting in a bimodal distribution of fragments. The same DNA samples were analyzed using the novel telomere assay, and the T/Q ratios were obtained as described earlier. The ratios varied between 0.1 and 2.2 for samples spanning TRFL values between $3.2-24.4 \mathrm{~kb}$, and there was good correlation between the two data sets $\left(\mathrm{R}^{2}=0.96\right)$. Notably, only the Jurkat DNA sample showed significant variation from the regression line.

\section{DISCUSSION}

The novel telomere assay described here is a rapid, reproducible, small-scale assay that measures telomere repeat se- quence content (TRSC) and normalizes the measurement for DNA input in a manner analogous to DNA indexing in flowFISH measurements of TRSC (9). The specificity of the assay was achieved through the use of oligonucleotides that are complementary to the known vertebrate telomere repeat sequence. In the absence of probe, we noted small sample-to-sample variations in the amount of background activity. There are at least three possible sources of background associated with the DNA: $(i)$ polymerase binding sites present at the free ends of linear genomic DNA molecules (this seems unlikely because telomeres terminate with single-stranded $3^{\prime}$ overhangs that are not substrates for the polymerase); (ii) polymerase binding sites within chromosomes because of sustained breaks or nicks; or (iii) nonspecific activity created as a result of unusual secondary structures generated after the denaturing step and upon renaturation. To account for background activity, we developed a mismatched probe that failed to initiate pyrophosphorolysis. In competition experiments using a half-maximal telo-probe concentration $(133 \mathrm{pM})$, titration of the control probe resulted in a dose-dependent inhibition of ATP production (data not shown). This confirmed the $3^{\prime}$ terminal specificity of the reaction and provided important evidence that the two probes bind to the same target sequences. Thus, the control probe reaction provided a useful means of subtracting background activity associated with the DNA quality and denaturation/renaturation effects, as well as possible oligonucleotide-
A

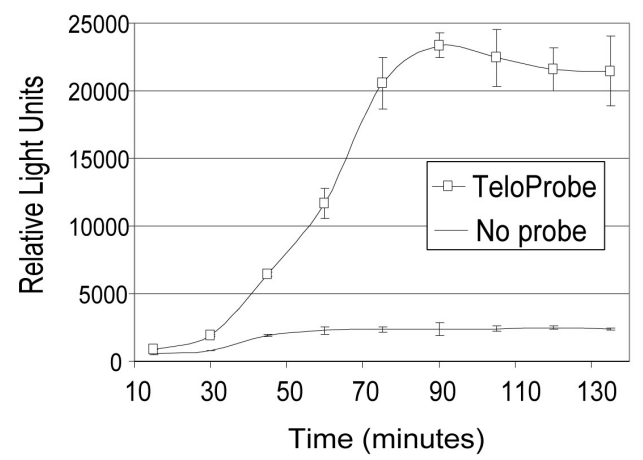

C

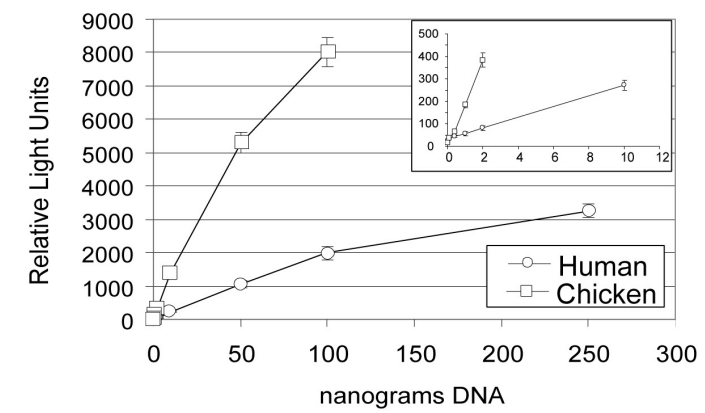

B

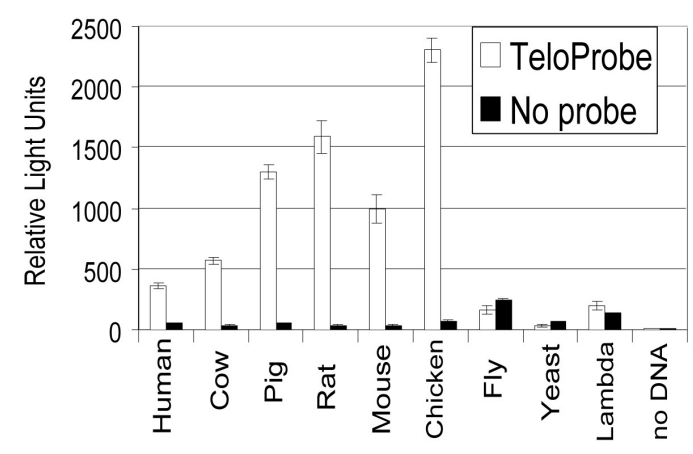

D

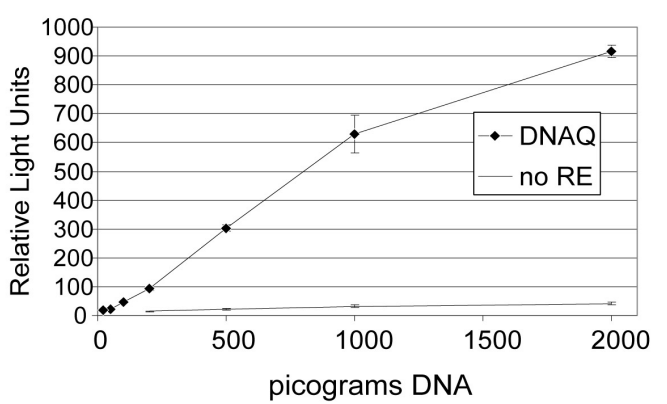

Figure 2. Telo-Glo Assay specificity and sensitivity, and DNA quantitation. (A) Ten nanograms of human DNA were arrayed and denatured in multiple wells of a PCR plate, and pyrophosphorolysis was carried out in the presence (squares) or absence (unmarked line) of the telo-probe. Reactions were performed in triplicate. Aliquots were removed at timed intervals, $50 \mu \mathrm{L}$ luciferin/luciferase detection reagent were added, and the luminescent detection of ATP was performed. (B) Ten nanograms of DNA from human, cow, pig, rat, mouse, chicken, fruit fly, yeast, or $\lambda$ were assayed using the telo-probe (open bars) or no probe (black bars) as illustrated. (C) Subsequently, telo-probe reactions were performed with 0-250 ng human (circles) or chicken (squares) DNA and a fixed amount of telo-probe $(3.3 \mu \mathrm{M})$. The inset graph shows data from the lower end of each curve. Each assay was performed in triplicate. Error bars represent standard deviations $(n=3)$. (D) Human DNA (0-2 ng) was incubated in the absence of oligonucleotides with a master mixture containing $1 \mathrm{U}$ restriction enzyme mixture per reaction (squares) or no restriction enzymes (unmarked line). The reactions were incubated at $37^{\circ} \mathrm{C}$ for 30 min and then transferred to $55^{\circ} \mathrm{C}$ for an additional $1 \mathrm{~h}$. Each assay was performed in triplicate. The error bars represent standard deviations $(n=3)$. 
dependent effects on the polymerase and kinase activities.

TRFL analysis generates an estimate of the mean length of telomeres within a distribution of fragment sizes. We used six separate human cell lines in an attempt to correlate our assay results to TRFL by using a commercially available Southern blot assay (TeloTAGGG; Roche Applied Science) and a digital imaging algorithm. For a DNA sample with a Gaussian distribution of telomere fragments, one predicts that the mean TRFL $=$ the modal TRFL $=$ median TRFL. However, we rarely observed normal distributions. In most cases, the histograms appeared to be skewed toward smaller telomere fragments, and the mean, mode, and median values were not equivalent. Thus, the mean TRFL value alone may not best reflect the TRSC of a cell. Furthermore, it has been noted that TRFL estimates in cells with short telomeres, such as those in the HL60 cell line, may represent an overestimate because the Southern blot method used here cannot account for the contribution of sub-telomeric sequences that may be as much as 3 $\mathrm{kb}$ in length (9). Such overestimates, combined with the assumption that our assay is not affected by sub-telomeric sequence or non-Gaussian distributions, would explain why the regression line in Figure 3B intercepts the $\mathrm{x}$-axis at $>0$. The estimated TRFL value of the Jurkat sample was also drawn into question because of the unusual bimodal distribution of fragments and its variance from the regression line. Despite the inherent difficulties comparing the mean telomere length

A $\begin{array}{llllll}1 & 2 & 3 & 4 & 5 & 6\end{array}$

B
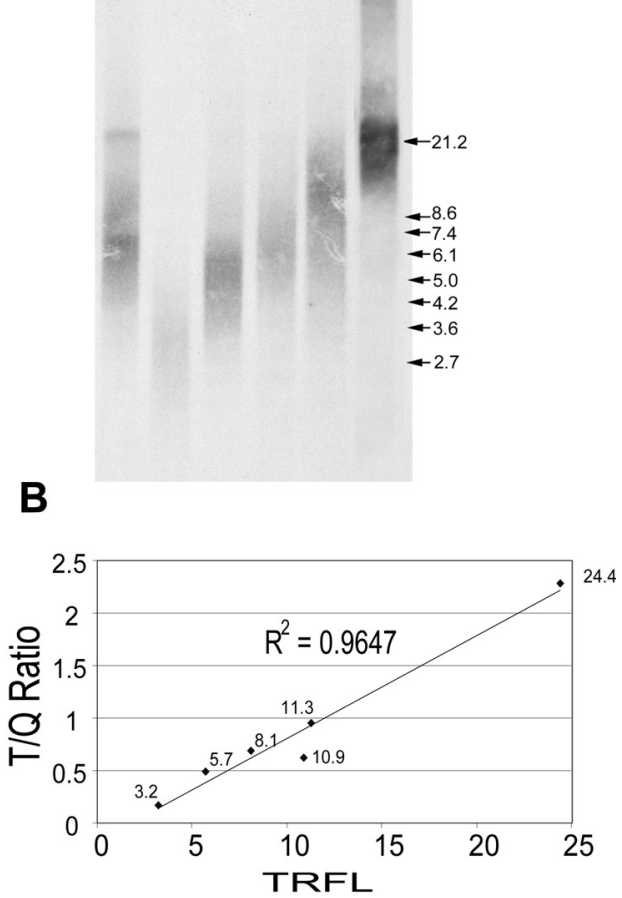

Figure 3. Correlation between TRFL analysis and the Telo-Glo Assay. (A) Six human cell lines were grown in culture, DNA was isolated, and TRFL analysis was performed. Molecular weight markers (arrows) are in kb. The DNA in lanes 1-6 are: Jurkat, HL60, SH-SY5Y, HeLa, HEK293, and HeLa subclone 1.2.11, respectively. (B) Ten nanograms of each DNA was assayed in triplicate using both the telo-probe and the control probe, and 500 or $0 \mathrm{pg}$ (blank) of DNA was used as input for the DNA quantitation reaction. The T/Q ratios were calculated and plotted against the corresponding TRFL values. Data points are labeled with TRFL values. to the TRSC, we observed a highly significant correlation between TRFL and T/Q ratios with an $\mathrm{R}^{2}$ value of 0.96 .

Since this novel assay has been adapted for use in a 96-well format, can be performed in approximately $4 \mathrm{~h}$, and requires only small amounts of DNA input, it offers a significant advantage in ease of use compared to other methods. Most of the data employed the use of $10 \mathrm{ng}$ DNA, but the assay is sensitive to less than $1 \mathrm{ng}$ or the equivalent of approximately 300 cells. This raises new possibilities in basic and clinical research, such as monitoring global changes to telomeres in small colonies expanded from single cells in vitro or limiting numbers of cells isolated by FACS ${ }^{\circledR}$ (BD Biosciences, San Jose, CA, USA). The assay might also serve as a measure of the replicative age of primary stem cells, and thus a qualitycontrol assay for the banking of such cells. Future studies to define additional performance criteria such as resolving power and reproducibility between laboratories are expected to further enhance the usefulness of the Telo-Glo assay.

\section{REFERENCES}

1.Blackburn, E.H. 2000. Telomere states and cell fates. Nature 408:53-56.

2.Delany, M.E., A.B. Krupkin, and M.M. Miller. 2000. Organization of telomere sequences in birds: evidence for arrays of extreme length and for in vivo shortening. Cytogenet. Cell Genet. 90:139-145.

3.Gharizadeh, B., T. Nordström, A. Ahmadian, M. Ronaghi, and P. Nyrén. 2002. Long-read pyrosequencing using pure 2'-deoxyadenosine5'-O'-(1-thiotriphosphate) Sp-isomer. Anal. Biochem. 301:10.1006/ abio.2001.5494.

4.Grant, J.D., D. Broccoli, M. Muquit, F.J. Manion, J. Tisdall, and M.F. Ochs. 2001. Telometric: a tool providing simplified, reproducible measurements of telomeric DNA from constant field agarose gels. BioTechniques 31:1314-1318.

5.Hultdin, M., E. Gronlund, K. Norrback, E. Eriksson-Lindstrom, T. Just, and G. Roos. 1998. Telomere analysis by fluorescence in situ hybridization and flow cytometry. Nucleic Acids Res. 26:3651-3656.

6.Karlseder, J., D. Broccoli, Y. Dai, S. Hardy, and T. de Lange. 1999. p53- and ATM-dependent apoptosis induced by telomeres lacking TRF2. Science 283:1321-1325.

7.Lansdorp, P.M., N.P. Verwoerd, F.M. van de Rijke, V. Dragowska, M.T. Little, R.W. Dirks, A.K. Raap, and H.J. Tanke. 1996. Heterogeneity in telomere length of human chromosomes. Hum. Mol. Genet. 5:685-691.

8.Mandrekar, M.N., A.M. Erickson, K. Kopp, B.E. Krenke, P.V. Mandrekar, R. Nelson, K. Peterson, J. Shultz, et al. 2001. Development of a Human DNA quantitation system. Croat. Med. J. 42:336-339.

9.Rufer, N., W. Dragowska, G. Thornbury, E. Roosnek, and P.M. Lansdorp. 1998. Telomere length dynamics in human lymphocyte subpopulations measured by flow cytometry. Nat. Biotechnol. 16:743-747.

10.Ryan, A., R. Schifreen, J. Shultz, I. MacIver, and D. Kephart. 2000. Technologies for mutation detection, IVD Technology, July/August Supplement.

11.Sambrook, J., E.F. Fritsch, and T. Maniatas. 1989. Molecular Cloning, A Laboratory Manual, 2nd edition. CSH Laboratory Press, Cold Spring Harbor, NY.

12.Schultz, J.W., M.K. Lewis, D.M. Leippe, M. Mandrekar, C.A. Andrews, J.R. Harnett, and R. Welch, inventors; Promega, assignee. Depolymerization method for nucleic acid detection of an amplified nucleic acid target. US patent 6277 578. 2001 Aug 21.

13.Schultz, J.W., M. Mandrekar, D.M. Leippe, M.K. Lewis, and L.S. Nelson, inventors; Promega, assignee. Nucleic acid detection. US patent 6159693. 2000 Dec 12.

14.Van Steensel, B. and T. DeLange. 1997. Control of telomere length by the human telomeric protein TRF1. Nature 385:740-743.

Address correspondence to Dr. Randall D. Learish, Promega, 2800 Woods Hollow Road, Madison, WI 53711, USA.e-mail: rlearish@promega.com 